\title{
MULTIVARIATE INTERPOLATION AND CONDITIONALLY POSITIVE DEFINITE FUNCTIONS. II
}

\author{
W. R. MADYCH AND S. A. NELSON
}

\begin{abstract}
We continue an earlier study of certain spaces that provide a variational framework for multivariate interpolation. Using the Fourier transform to analyze these spaces, we obtain error estimates of arbitrarily high order for a class of interpolation methods that includes multiquadrics.
\end{abstract}

\section{INTRODUCTION}

This paper continues a study, [11], of certain subspaces $C_{h}$ of $C\left(\mathbf{R}^{n}\right)$, the continuous complex-valued functions on $n$-space $\mathbf{R}^{n}$. The spaces $C_{h}$ provide a variational framework for the following interpolation problem: given numerical values at a scattered set of points in $\mathbf{R}^{n}$, make a good choice of a function $f$ in $C\left(\mathbf{R}^{n}\right)$ that takes on those values.

For the reader's convenience we review some basic features of the development in [11]. The starting point is the selection of an integer $m \geq 0$ and a continuous function $h$ on $\mathbf{R}^{n}$ that is conditionally positive definite of order $m$. For example: $m=1, h(x)=-\sqrt{1+|x|^{2}}$. Using $h$, a space $C_{h}$ with a semi-inner product $(\cdot, \cdot)_{h}$ is constructed. $C_{h}$ is a subspace of $C\left(\mathbf{R}^{n}\right)$, and the null space of $(\cdot, \cdot)_{h}$ is $P_{m-1}$, the polynomials on $\mathbf{R}^{n}$ of degree $m-1$ or less. A key property of $C_{h}$ is this: if $x_{1}, \ldots, x_{N}$ are distinct points in $\mathbf{R}^{n}$ and $v_{1}, \ldots, v_{N}$ are complex numbers, then among all functions $f$ in $C_{h}$ that satisfy the interpolation conditions $f\left(x_{i}\right)=v_{i}$, the quadratic $\|f\|_{h}^{2}=(f, f)_{h}$ is minimized by a function of the form $f=s+p$, where $p$ is in $P_{m-1}$ and

$$
s(x)=\sum_{i=1}^{N} c_{i} h\left(x-x_{i}\right)
$$

with $\sum_{i=1}^{N} c_{i} x_{i}^{k}=0$ for all $|\alpha|<m$. For the example mentioned, (1.1) is a multiquadric interpolant.

Because the spaces $C_{h}$ are translation-invariant, the Fourier transform is a natural tool for analyzing them; it plays a central role here. To clarify basic ideas and make an orderly division of our results, we avoided Fourier techniques in

Received December 8, 1988.

1980 Mathematics Subject Classification (1985 Revision). Primary 41A05, 41A63.

Both authors were partially supported by a grant from the Air Force Office of Scientific Research, AFOSR-86-0145. 
[11]. We did, however, rely on them in our earlier investigation [10], which was in fact prompted by the Fourier methods in Duchon [5]. Use of Fourier transforms allows us to give improved descriptions of the spaces $C_{h}$ (see $\S 3$ ) and allows us to single out certain cases where error estimates of order $l \geq m$ are possible (see $\S 4$ ). These estimates apply to the multiquadric case as well as to related examples given in $\S 5$; for each example given there, the integer $l$ can be arbitrarily large.

\section{Preliminaries}

In this section we recall some notation and results involving Fourier transforms and conditionally positive definite functions.

Let $\mathscr{D}\left(\mathbf{R}^{n}\right)$ denote the space of complex-valued functions on $\mathbf{R}^{n}$ that are compactly supported and infinitely differentiable. The Fourier transform of a function $\varphi$ in $\mathscr{D}$ is

$$
\widehat{\varphi}(\xi)=\int e^{-i\langle x, \xi\rangle} \varphi(x) d x
$$

In order to make use of theorems from Gelfand and Vilenkin [7], we adopt their definition of $m$ th-order conditional positive definiteness. (Equivalence with the definition used in [11] can be seen from Proposition 2.4 and Theorem 6.1 below.) Thus, for a continuous function $h$ we assume

$$
\int h(x) \varphi * \tilde{\varphi}(x) d x \geq 0
$$

holds whenever $\varphi=p(D) \psi$ with $\psi$ in $\mathscr{D}$ and $p(D)$ a linear homogeneous constant coefficient differential operator of order $m$. Here $\tilde{\varphi}(x)=\overline{\varphi(-x)}$ and * denotes the convolution product

$$
\varphi_{1} * \varphi_{2}(t)=\int \varphi_{1}(x) \varphi_{2}(t-x) d x
$$

Note that (2.2) can be rewritten as

$$
\iint h(x-y) \varphi(x) \overline{\varphi(y)} d x d y \geq 0 \text {. }
$$

The following result can be found in Chapter II, Section 4.4 of [7]; we incorporate a remark at the end of that section concerning the case where $h$ is continuous.

Theorem 2.1. Let $h$ be continuous and conditionally positive definite of order $m$. Then it is possible to choose a positive Borel measure $\mu$ on $\mathbf{R}^{n} \sim\{0\}$, constants $a_{, \prime},|\gamma| \leq 2 m$ and a function $\chi$ in $\mathscr{D}$ such that: $1-\hat{\chi}(\xi)$ has a zero of order $2 m+1$ at $\xi=0 ;$ both of the integrals $\int_{0<|\xi|<1}|\xi|^{2 m} d \mu(\xi), \int_{|\xi| \geq 1} d \mu(\xi)$ are finite; for all $\psi \in \mathscr{Z}$,

$$
\begin{aligned}
\int h(x) \psi(x) d x= & \int\left[\widehat{\psi}(\xi)-\widehat{\chi}(\xi) \sum_{\left|\gamma^{\prime}\right|<2 m} D^{\prime} \widehat{\psi}(0) \frac{\xi^{\prime}}{\gamma^{\prime}}\right] d \mu(\xi) \\
& +\sum_{|;| \leq 2 m} D^{\prime \prime} \widehat{\psi}(0) \frac{a_{\eta^{\prime}}}{\gamma !} .
\end{aligned}
$$


This uniquely determines the measure $\mu$ and the constants $a_{\gamma}$ for $|\gamma|=2 m$. In addition, for every choice of complex numbers $c_{\alpha},|\alpha|=m$,

$$
\sum_{|\alpha|=m|\beta|=m} \sum_{\alpha+\beta} c_{\alpha} \bar{c}_{\beta} \geq 0
$$

The choice of $\chi$ affects the value of the coefficients $a_{y}$ for $|\gamma|<2 m$. Note that the value of the right side of (2.4) does not change if, for suitable $\varphi, \hat{\chi}$ is replaced by $\hat{\chi}+\varphi$ and the $a_{\gamma}$, for $|\gamma|<2 m$, are replaced by $a_{\gamma}+\int \varphi(\xi) \xi^{\prime} d \mu(\xi)$.

As can be seen from

$$
(-i)^{|i|} \int x^{i} \varphi(x) d x=D^{\gamma} \hat{\varphi}(0)
$$

changing a coefficient $a_{\gamma}$, on the right-hand side of (2.4) corresponds to changing $h(x)$ on the left side by adding a constant multiple of $x^{y}$.

For $m=0,(2.4)$ reduces to $\int h \psi=\int \widehat{\psi} d \lambda$, where $\lambda$ is the Borel measure on $\mathbf{R}^{n}$ given by

$$
\lambda(E)=\mu(E \sim\{0\})+a_{0} \delta(E) .
$$

Here $\delta$ is the measure corresponding to a unit mass at the origin; $\delta(E)=1$ if $0 \in E$ and $\delta(E)=0$ otherwise. Recall that Borel measures that are finite on compact sets are called Radon measures. We make the usual identification of a Radon measure on an open set $\Omega \subset \mathbf{R}^{n}$ with the corresponding distribution in $\mathscr{D}^{\prime}(\Omega)$ and write $\langle\lambda, \psi\rangle=\int \psi d \lambda$. Also, if $f \in L_{\text {loc }}^{1}\left(\mathbf{R}^{n}\right)$, we identify it with the distribution in $\mathscr{D}^{\prime}$ given by $\langle f, \psi\rangle=\int \psi(x) f(x) d x$. Thus, for $m=0$, (2.4) says $\langle h, \varphi\rangle=\langle\lambda, \widehat{\varphi}\rangle$.

For an illustration of the theorem when $m \neq 0$, take $n=2, m=1$, $h(x)=-\sqrt{1+|x|^{2}}$. Then $d \mu(\xi)=w(\xi) d \xi$ with

$$
w(\xi)=\frac{(1+|\xi|) e^{-|\xi|}}{(2 \pi)^{2}|\xi|^{3}}
$$

and $a_{\gamma}=0$ for $|\gamma|=2$. If $\chi$ is even, then the coefficients $a_{\gamma}$ for $|\gamma|=1$ are also 0 . The remaining coefficient is $a_{0}=-\left(1+\int[1-\widehat{\chi}(\xi)] w(\xi) d \xi\right)$. Details for this and related examples are given in $\S 5$.

We use $T^{k} \varphi$ to denote the $k$ th-order Taylor polynomial for $\varphi$ about 0 :

$$
T^{k} \varphi(\xi)=\sum_{|\alpha| \leq k} D^{\alpha} \varphi(0) \frac{\xi^{\alpha}}{\alpha !} .
$$

The integral on the right side of (2.4) can then be written as $\int \widehat{\psi}-\widehat{\chi} T^{2 m-1} \widehat{\psi} d \mu$.

The Schwartz space of rapidly decreasing $C^{\infty}$ functions and its dual, the space of tempered distributions, are denoted by the usual letters $\mathscr{S}$ and $\mathscr{S}^{\prime}$.

Proposition 2.2. Let $k$ be a positive integer and let $\sigma$ be a Radon measure on $\mathbf{R}^{n} \sim\{0\}$ such that $\int|\xi|^{k}\left(1+|\xi|^{k}\right)^{-1} d|\sigma|(\xi)<\infty$. Let $s$ be a continuous 
function such that $|\xi|^{k} s(\xi)$ is bounded on $\mathbf{R}^{n}$ and $1-s(\xi)=O\left(|\xi|^{k}\right)$ at $\xi=0$. Let

$$
u(x)=\int\left[e^{-i\langle x, \xi\rangle}-s(\xi) \sum_{r=0}^{k-1} \frac{(-i\langle x, \xi\rangle)^{r}}{r !}\right] d \sigma(\xi)
$$

Then $u \in C\left(\mathbf{R}^{n}\right), u(x)=o\left(|x|^{k}\right)$ as $|x| \rightarrow \infty$ and for all $\varphi$ in $\mathscr{S}$

$$
\int u(x) \varphi(x) d x=\int\left(\widehat{\varphi}-s T^{k-1} \hat{\varphi}\right) d \sigma .
$$

Proof. Let $E(t)=e^{-i t}-\sum_{r=0}^{k-1}(-i t)^{r} / r$ ! and note that $u=u_{0}$, where

$$
u_{a}(x)=\int_{|\xi|>a}(1-s(\xi)) e^{-i\langle x, \xi\rangle}+s(\xi) E(\langle x, \xi\rangle) d \sigma(\xi) .
$$

From $|E(t)| \leq|t|^{k}$ we have $|s(\xi) E(\langle x, \xi\rangle)| \leq|x|^{k}|\xi|^{k}|s(\xi)|$. Our assumptions on $\sigma$ and $s$ ensure that $1-s(\xi)$ and $|\xi|^{k}|s(\xi)|$ belong to $L^{1}(\sigma)$. Continuity of $u$ can be established using dominated convergence.

To prove $u(x)=o\left(|x|^{k}\right)$, note that $\left|u_{0}(x)-u_{a}(x)\right| \leq\left(c_{1}(a)+c_{2}(a)|x|^{k}\right)$, where $c_{1}(a)$ and $c_{2}(a)$ are the results of integrating $|1-s(\xi)|$ and $|\xi|^{k}|s(\xi)|$ over $0<|\xi| \leq a$ with respect to $|\sigma|$. Given $\varepsilon>0$, choose $a>0$ so that $c_{1}(a)<\varepsilon$ and $c_{2}(a)<\varepsilon$. From $|E(t)| \leq 2|t|^{k-1}$ and $a>0$ we have $u_{a}(x)=O\left(|x|^{k-1}\right)$ as $|x| \rightarrow \infty$. Thus, we may choose $R \geq 1$ such that $\left|u_{a}(x)\right| \leq \varepsilon|x|^{k}$ for all $|x|>R$. Then, for $|x|>R$,

$$
|u(x)| \leq\left|u_{a}(x)\right|+\left|u_{0}(x)-u_{a}(x)\right| \leq \varepsilon|x|^{k}+\varepsilon+\varepsilon|x|^{k} .
$$

It follows that $u(x)=o\left(|x|^{k}\right)$.

To establish (2.9), apply Fubini's theorem and use

$$
\int \frac{(-i\langle x, \xi\rangle)^{r}}{r !} \varphi(x) d x=\sum_{|\alpha|=r} D^{\alpha} \widehat{\varphi}(0) \frac{\xi^{\prime \prime}}{\alpha !} .
$$

This can be verified by using $\left(y_{1}+\cdots+y_{n}\right)^{r} / r !=\sum_{|\alpha|=r} y^{\alpha x} / \alpha !$ and (2.6).

If $u$ is defined by (2.8) with $\sigma=\mu, k=2 m$ and $s=\hat{\chi}$, then from (2.4), (2.9) and (2.6) we have $\langle h-u, \psi\rangle=\langle q, \psi\rangle$ for all $\psi$ in $\mathscr{D}$. Here, $q(x)=\sum_{\left|y^{\prime}\right| \leq 2 m} a_{y}(-i x)^{\eta^{\prime}} / \gamma !$.

Corollary 2.3. Suppose $h$ is continuous and positive definite of order $m$. If $m>0$, then there are unique constants $a_{y,},|\gamma|=2 m$, such that

$$
h(x)-\sum_{|;|=2 m} a_{i,}(-i x)^{\gamma} / \gamma !=o\left(|x|^{2 m}\right), \quad \text { as }|x| \rightarrow \infty .
$$

These constants are the same as those appearing in (2.4).

For ease in dealing with (2.5), we develop some related notation. Let $V_{m}$ be the space of vectors $v=\left(v_{\alpha}\right)_{|\alpha|=m}$ and let $A$ be the operator on $V_{m}$ defined 
by $A v=w$ where $w_{\alpha}=\sum_{|\beta|=m} A_{\alpha, \beta} v_{\beta}$ and $A_{\alpha, \beta}=a_{\alpha+\beta} /(\alpha ! \beta !)$. Because of (2.5), $A$ must be real-symmetric. Thus $A v=0$ if and only if $v^{T} \overline{A v}=0$. Equivalently, the null space, $N_{A}$, of $A$ is the null space of the semi-inner product $(v, w)_{A}=v^{T} \overline{A w}$. Let $H_{A}=V_{m} / N_{A}$ be the Hilbert space obtained by identifying $v$ and $w$ whenever $\|v-w\|_{A}=0$. The elements of $H_{A}$ are the cosets $v+N_{A}$, and as $w$ varies over such a coset, $A w$ remains fixed.

By applying Theorem 2.1 we can recover (2.2) for a more convenient set of functions $\varphi$. Let

$$
\mathscr{D}_{m}=\left\{\varphi \in \mathscr{D}: \int x^{\alpha} \varphi(x) d x=0 \text { for all }|\alpha|<m\right\} .
$$

Clearly, $\mathscr{D}_{m}=\left\{\varphi \in \mathscr{D}: \widehat{\varphi}(\xi)=O\left(|\xi|^{m}\right)\right.$ at $\left.\xi=0\right\}$. If $\psi=\varphi * \tilde{\varphi}$, then $\widehat{\psi}=|\widehat{\varphi}|^{2}$, so

$$
D^{\gamma} \widehat{\psi}=\sum_{\alpha+\beta=\gamma} \frac{\gamma !}{\alpha ! \beta !} D^{\alpha} \hat{\varphi} D^{\beta} \overline{\widehat{\varphi}} .
$$

Hence, for $\psi=\varphi * \tilde{\varphi}$ with $\varphi \in \mathscr{D}_{m}$,

$$
\sum_{|:| \leq 2 m} D^{\gamma} \widehat{\psi}(0) \frac{a_{\gamma}}{\gamma !}=\sum_{|\alpha|=m|\beta|=m} \sum_{k+\beta} \frac{D^{\alpha} \hat{\varphi}(0)}{\alpha !} \frac{D^{\beta} \overline{\hat{\varphi}(0)}}{\beta !}=\left\|\hat{\varphi}^{(m)}(0)\right\|_{A}^{2},
$$

where $\hat{\varphi}^{(m)}(0)$ is the vector $v$ in $V_{m}$ given by $v_{\alpha}=D^{\alpha} \widehat{\varphi}(0)$. From (2.4) we see that if $\varphi \in \mathscr{D}_{m}$, then

$$
\int h(x) \varphi * \tilde{\varphi}(x) d x=\int|\widehat{\varphi}|^{2} d \mu+\left\|\widehat{\varphi}^{(m)}(0)\right\|_{A}^{2},
$$

and (2.2) holds. Since $\mathscr{D}_{m}$ includes the functions $\varphi$ for which (2.2) was assumed, we conclude that requiring (2.2) for all $\varphi \in \mathscr{D}_{m}$ is an equivalent definition of $h$ being conditionally positive definite of order $m$.

Since $\mathscr{D}_{m+1} \subset \mathscr{D}_{m}$, the latter definition makes it clear that $h$ will be conditionally positive definite of order $m+1$ if it is conditionally positive definite of order $m$. If $m$ is replaced by $m+1$ in Theorem 2.1 , with $h$ held fixed, the measure $\mu$ will remain the same, the coefficients $a_{\gamma,},|\gamma|=2(m+1)$, will be 0 , and the lower-order coefficients will change to reflect changes in $\hat{\chi}$ and additional terms in the Taylor polynomial.

In order to apply results from [11], we verify that $h$ is in the space $Q_{m}\left(\mathbf{R}^{n}\right)$ defined there.

Proposition 2.4. Let $h$ be continuous and assume (2.2) holds for all $\varphi \in \mathscr{D}_{m}$. If $x_{1}, \ldots, x_{N}$ are distinct points in $\mathbf{R}^{n}$ and $c_{1}, \ldots, c_{N}$ are constants that satisfy $\sum_{i=1}^{N} c_{i} x_{i}^{\prime \prime}=0$ for all $|\alpha|<m$, then

$$
\sum_{i, j=1}^{N} c_{i} \bar{c}_{j} h\left(x_{i}-x_{j}\right) \geq 0 \text {. }
$$

Proof. Choose $g$ in $\mathscr{D}$ with $\int g(x) d x=1$ and $g(x)=0$ for all $|x| \geq 1$. For $\varepsilon>0$, let $g_{\varepsilon}=\varepsilon^{-n} g(x / \varepsilon)$ and take $\varphi_{\varepsilon}(x)=\sum_{k=1}^{N} c_{k} g_{\varepsilon}\left(x-x_{k}\right)$. Then 
$\widehat{\varphi_{\varepsilon}}(\xi)=\tau(\xi) \widehat{g}(\varepsilon \xi)$ with $\tau(\xi)=\sum_{k=1}^{N} c_{k} e^{-i\left\langle x_{k}, \xi\right\rangle}$. From

$$
D^{\alpha} \tau(\xi)=\sum_{k=1}^{N} c_{k}\left(-i x_{k}\right)^{a} e^{-i\left\langle x_{k}, \xi\right\rangle}
$$

we find $\tau(\xi)=O\left(|\xi|^{m}\right)$ at $\xi=0$. Thus $\varphi_{\varepsilon} \in \mathscr{D}_{m}$ and

$$
0 \leq \int h(x) \varphi_{\varepsilon} * \tilde{\varphi}_{\varepsilon}(x) d x=\iint h(t-y) \varphi_{\varepsilon}(t) \overline{\varphi_{\varepsilon}(y)} d t d y .
$$

Letting $\varepsilon \rightarrow 0$, we obtain (2.13).

The following observations will be used in the next section. Let $\widehat{\mathscr{D}}_{m}=$ $\left\{\widehat{\varphi}: \varphi \in \mathscr{D}_{m}\right\}$.

Proposition 2.5. Let $m \geq 0$ and let $\mu$ be a positive Borel measure on $\mathbf{R}^{n} \sim\{0\}$ that satisfies $\int\left(|\xi|^{m} /\left(1+|\xi|^{m}\right)\right)^{2} d \mu(\xi)<\infty$. If $2 k \geq m$, then $\widehat{\mathscr{D}}_{2 k}$ is a dense subset of $L^{2}(\mu)$.

Proof. Let $g \in L^{2}(\mu)$ and $\varepsilon>0$. Choose $g_{1} \in \mathscr{D}\left(\mathbf{R}^{n} \sim\{0\}\right)$ so that $\left\|g-g_{1}\right\|_{L^{2}(\mu)}<\varepsilon$. Then $f(\xi)=|\xi|^{-2 k} g_{1}(\xi)$ is in $\mathscr{D}$. Since $\widehat{\mathscr{D}}$ is dense in $\mathscr{P}$, we can find $\psi \in \mathscr{D}$ so that for all $\xi$ in $\mathbf{R}^{n},|f(\xi)-\widehat{\psi}(\xi)| \leq \varepsilon /\left(1+|\xi|^{2 k}\right)$. Multiplying by $|\xi|^{2 k}$ gives

$$
\left.\left|g_{1}(\xi)-\right| \xi\right|^{2 k} \widehat{\psi} \mid \leq \frac{\varepsilon|\xi|^{2 k}}{1+|\xi|^{2 k}} .
$$

Let $\varphi=(-\Delta)^{k} \psi$. Then $\varphi \in \mathscr{D}, \widehat{\varphi}(\xi)=|\xi|^{2 k} \widehat{\psi}(\xi)$ and

$$
\int\left|g_{1}-\widehat{\varphi}\right|^{2} d \mu \leq \varepsilon^{2} \int\left(\frac{|\xi|^{2 k}}{1+|\xi|^{2 k}}\right)^{2} d \mu(\xi) \text {. }
$$

Thus $\|g-\hat{\varphi}\|_{L^{2}(\mu)}$ can be made as small as desired with $\varphi \in \mathscr{D}_{2 k}$.

Proposition 2.6. If $T \in \mathscr{D}^{\prime}$ satisfies $T(\varphi)=0$ for all $\varphi$ in $\mathscr{D}_{m}$, then $T$ belongs to $P_{m-1}$.

Proof. Define $T_{\alpha} \in \mathscr{D}^{\prime}$ by $T_{\alpha}(\varphi)=\int x^{\alpha} \varphi(x) d x$ and note that $\cap\left\{T_{\alpha}^{-1}(0)\right.$ : $|\alpha|<m\}=\mathscr{D}_{m}$. By assumption, $\mathscr{D}_{m}$ is contained in $T^{-1}(0)$, the null space of $T$. It follows (see Theorem 1.3 of [9]) that there are constants $c_{\alpha}$ such that $T=\sum_{|\alpha|<m} c_{\alpha} T_{\alpha}$.

\section{FOURIER DESCRIPTION OF $C_{h}$}

After analyzing the space $\mathscr{C}_{h, m}$ defined below, we will see that it coincides with the space $C_{h}$ studied in [11]. Among the results emerging from this analysis is a Fourier transform description of $\mathscr{C}_{h, m}$. 
Definition. Let $h$ be a continuous function on $\mathbf{R}^{n}$ that is conditionally positive definite of order $m$. We write $f \in \mathscr{C}_{h, m}\left(\mathbf{R}^{n}\right)$ if $f \in C\left(\mathbf{R}^{n}\right)$ and there is a constant $c(f)$ such that for all $\varphi$ in $\mathscr{D}_{m}$

$$
\left|\int f(x) \varphi(x) d x\right| \leq c(f)\left\{\iint h(x-y) \varphi(x) \overline{\varphi(y)} d x d y\right\}^{1 / 2}
$$

If $f \in \mathscr{C}_{h, m}\left(\mathbf{R}^{n}\right)$ we let $c_{*}(f)$ denote the smallest constant for which (3.1) is true.

It is easily checked that if $f_{1}$ and $f_{2}$ are in $\mathscr{C}_{h, m}$, then $f_{1}+f_{2}$ and $a f_{1}, a \in$ C, are also in $\mathscr{C}_{h, m}$ with $c_{*}\left(f_{1}+f_{2}\right) \leq c_{*}\left(f_{1}\right)+c_{*}\left(f_{2}\right)$ and $c_{*}\left(a f_{1}\right)=|a| c_{*}\left(f_{1}\right)$. If $f \in P_{m-1}$ and $\varphi \in \mathscr{D}_{m}$, then $\langle f, \varphi\rangle=0$, so $f \in \mathscr{C}_{h, m}$ and $c_{*}(f)=0$. Conversely, if $c_{*}(f)=0$, then $f \in P_{m-1}$ by Proposition 2.6. Thus $c_{*}(f)$ is a seminorm with null space $P_{m-1}$; for $m=0$, take $P_{-1}=\{0\}$.

Using (2.12), we note that (3.1) is equivalent to

$$
|\langle f, \varphi\rangle| \leq c(f)\left\{\|\widehat{\varphi}\|_{L^{2}(\mu)}^{2}+\left\|\hat{\varphi}^{(m)}(0)\right\|_{A}^{2}\right\}^{1 / 2}
$$

for all $\varphi$ in $\mathscr{D}_{m}$. If $v \in V_{m}$ and

$$
q(x)=\sum_{|\alpha|=m}(A v)_{\alpha}(-i x)^{\alpha}
$$

then $\langle q, \varphi\rangle=\sum_{|\alpha|=m}(A v)_{\alpha} D^{\alpha} \widehat{\varphi}(0)=\left(\widehat{\varphi}^{(m)}(0), \bar{v}\right)_{A}$, so $q \in \mathscr{C}_{h, m}$ with $c_{*}(q)=$ $\|\bar{v}\|_{A}$. If $g \in L^{2}(\mu)$ and $u$ is defined by (2.8) with $\sigma=g \mu, k=m$ and an appropriate choice of $s$ (take $s=0$ for $m=0$ ), then, for $\varphi \in \mathscr{D}_{m},(2.9)$ gives $\langle u, \varphi\rangle=\int \hat{\varphi} g d \mu$. It follows that $u \in \mathscr{C}_{h, m}$ with $c_{*}(u)=\|g\|_{L^{2}(\mu)}$.

Clearly, $\mathscr{C}_{h, m}$ includes all functions of the form $f=u+q+p$ with $u, q$ as above and $p \in P_{m-1}$. The next result, when combined with Proposition 2.6, shows that all functions in $\mathscr{C}_{h, m}$ can be obtained in this way.

From the behavior of $u(x)$ as $|x| \rightarrow \infty$, described by Proposition 2.2, we see that if $m>0$ and $f=u+q+p$, then $f(x)=o\left(|x|^{m}\right)$ is equivalent to $q=0($ or $A v=0)$. In any case,

$$
\mathscr{C}_{h, m}\left(\mathbf{R}^{n}\right) \subset\left\{f \in C\left(\mathbf{R}^{n}\right): f(x)=O\left(|x|^{m}\right) \text { as }|x| \rightarrow \infty\right\} .
$$

Proposition 3.1. Let $m, h, \mu$ and $a_{\gamma}$ be as in Theorem 2.1. If $f \in \mathscr{C}_{h, m}$, then there is a function $g \in L^{2}(\mu)$ and a vector $v \in V_{m}$ such that for all $\varphi$ in $\mathscr{D}_{m}$

$$
\langle f, \varphi\rangle=\int \hat{\varphi} g d \mu+\sum_{|\alpha|=m}(A v)_{\alpha} D^{\alpha} \widehat{\varphi}(0) .
$$

This uniquely determines $g$ and the coset $v+N_{A}$.

Proof. Define $J: \mathscr{D}_{m} \rightarrow H=L^{2}(\mu) \oplus H_{A}$ by $J \varphi=\widehat{\varphi} \oplus\left(\hat{\varphi}^{(m)}(0)+N_{A}\right)$. From (3.2) we see that $|\langle f, \varphi\rangle| \leq c_{*}(f)\|J \varphi\|_{H}$. From this we deduce that, if $J \varphi_{1}=J \varphi_{2}$, then $\left\langle f, \varphi_{1}\right\rangle=\left\langle f, \varphi_{2}\right\rangle$. It follows that there is a bounded linear functional $L$ on the image $J \mathscr{D}_{m}$ such that $L(J \varphi)=\langle f, \varphi\rangle$ for all $\varphi$ 
in $\mathscr{D}_{m}$. Since $H$ is a Hilbert space, we can choose $\bar{g} \oplus\left(\bar{v}+N_{A}\right)$ so that for all $\varphi$ in $\mathscr{D}_{m},\langle f, \varphi\rangle=\left(J \varphi, \bar{g} \oplus\left(\bar{v}+N_{A}\right)\right)_{H}$. This gives (3.5).

For uniqueness, we show that $J \mathscr{D}_{m}$ is dense in $H$. Let $g_{1} \in L^{2}(\mu), w \in V_{m}$ and $\eta>0$ be given. Take $2 k>m$ and use Proposition 2.5 to choose $\varphi_{1} \in \mathscr{D}_{2 k}^{m}$ with $\left\|g_{1}-\widehat{\varphi}_{1}\right\|_{L^{2}(\mu)}<\eta$. Note that $J \varphi_{1}=\widehat{\varphi}_{1} \oplus 0$ since $2 k>m$. Put $p(\xi) \stackrel{=}{=}$ $\sum_{|\alpha|=m} w_{\alpha} \xi^{\alpha} / \alpha$ ! and take $\chi \in \mathscr{D}$ so that $1-\hat{\chi}(\xi)=O\left(|\xi|^{m+1}\right)$ at $\xi=0$. Define $\psi_{\varepsilon} \in \mathscr{D}$ by $\widehat{\psi}_{\varepsilon}(\xi)=p(\xi) \widehat{\chi}\left(\varepsilon^{-1} \xi\right)$. Then $J \psi_{\varepsilon}=\widehat{\psi}_{\varepsilon} \oplus\left(w+N_{A}\right)$. Choosing $\varepsilon$ close enough to 0 , we have $\left\|\widehat{\psi}_{\varepsilon}\right\|_{L^{2}(\mu)}<\eta$. Then $\left\|g_{1}+\left(w+N_{A}\right)-J\left(\varphi_{1}+\psi_{\varepsilon}\right)\right\|_{H}<$ $2 \eta$.

If $f \in \mathscr{C}_{h, m}$, let $\Lambda f=g \oplus\left(v+N_{A}\right)$ be the point in $H=L^{2}(\mu) \oplus H_{A}$ determined by (3.5). Clearly, the resulting map $\Lambda: \mathscr{C}_{h, m} \rightarrow H$ is linear. That $\Lambda$ maps onto $H$ is evident from the remarks leading up to Proposition 3.1. From (3.2) and (3.5) we see that $c_{*}(f)=\|\Lambda f\|_{H}$. Note $\|\Lambda f\|_{H}=\left\{(f, f)_{h}\right\}^{1 / 2}=$ $\|f\|_{h}$, where $\left(f_{1}, f_{2}\right)_{h}=\left(\Lambda f_{1}, \Lambda f_{2}\right)_{H}$ is a semi-inner product for $\mathscr{C}_{h, m}$. There is a corresponding inner product on $\mathscr{C}_{h, m} / P_{m-1}$, which is then a Hilbert space isomorphic to $H$ under the quotient map associated with $\Lambda$.

The following provides a converse to Proposition 3.1 and clarifies how the Fourier transform relates $f$ to $g, v$ in (3.5).

Proposition 3.2. Let $m, h, \mu$ and $a_{y}$ be as in Theorem 2.1. Fix $g \in L^{2}(\mu)$, $v \in V_{m}$ and $f \in \mathscr{D}^{\prime}$. The following are equivalent:

(a) (3.5) holds for all $\varphi$ in $\mathscr{D}_{m}$;

(b) $f \in \mathscr{P}^{\prime}$ and for every $|\alpha|=m, \xi^{\alpha} F=\lambda_{\alpha}$, where $F$ is the inverse Fourier transform of $f$ and $\lambda_{c}$ is the Radon measure on $\mathbf{R}^{n}$ given by

$$
\lambda_{,}(E)=\int_{E \sim\{0\}} \xi^{\alpha} g(\xi) d \mu(\xi)+\alpha !(A v)_{\alpha} \delta(E) \text {. }
$$

When this is the case, $f \in \mathscr{C}_{h, m}, \Lambda f=g \oplus\left(v+N_{A}\right)$ and $(f, f)_{h}=\int|g|^{2} d \mu+$ $v^{T} \overline{A v}$.

Proof. Let $q$ be as in (3.3) and let $u$ be defined by (2.8) with $\sigma=g \mu, k=m$ and a choice of $s$ that satisfies the hypotheses of Proposition 2.2. If (a) holds, then $\langle f, \varphi\rangle=\langle u+q, \varphi\rangle$ for all $\varphi \in \mathscr{D}_{m}$. By Proposition 2.6, $f-(u+q)=$ $p \in P_{m-1}$. If $\widehat{F}=f$ and $\widehat{\psi}(\xi)=\xi^{k} \varphi(\xi)$, then

$$
\begin{aligned}
\left\langle\xi^{\prime \prime} F, \varphi\right\rangle & =\langle F, \widehat{\psi}\rangle=\langle f, \psi\rangle=\langle u, \psi\rangle+\langle q+p, \psi\rangle \\
& =\int\left(\widehat{\psi}-s T^{m-1} \widehat{\psi}\right) g d \mu+\sum_{|\propto| \leq m} b_{\alpha} D^{\prime \prime} \widehat{\psi}(0),
\end{aligned}
$$

where the constants $b_{r}$ are determined by $q+p(x)=\sum_{|r| \leq m} b_{a}(i x)^{\alpha}$. Thus,

$$
\left\langle\xi^{\alpha} F, \varphi\right\rangle=\int\left(\xi^{\alpha} \varphi(\xi)-0\right) g(\xi) d \mu(\xi)+\alpha !(A v)_{\alpha} \varphi(0),
$$


which establishes (b). To see that (b) implies (a), let $f_{1}=u+q$ with $u$ and $q$ as above. Then (3.7) holds for $F_{1}$, where $\widehat{F}_{1}=f_{1}$. Hence, $\xi^{(\alpha} F_{1}=\lambda_{\alpha}$. If (b) holds, then $\xi^{\alpha} F_{1}=\xi^{\alpha} F$ for all $|\alpha|=m$. This implies $F_{1}-F=\sum_{|\alpha|<m} b_{c \alpha} D^{\alpha} \delta$, which says $f_{1}-f \in P_{m-1}$. Therefore, (a) and the other assertions about $f$ follow from the corresponding facts about $f_{1}$.

For typical choices of $h$ (e.g. those considered in $\S 5$ ) the measure $\mu$ is absolutely continuous with respect to Lebesgue measure, $d \mu(\xi)=w(\xi) d \xi$, and $a_{\gamma}=0$ for all $|\gamma|=2 m$. In such cases the measures $\lambda_{\alpha}$ in (3.6) are given by functions $F_{c \alpha}$ in $L_{\text {loc }}^{1}\left(\mathbf{R}^{n}\right) ; d \lambda_{c}(\xi)=F_{c \alpha}(\xi) d \xi$, where $F_{c \alpha}(\xi)=\xi^{\alpha} g(\xi) w(\xi)$. From $D^{\alpha} f=\left((-i \xi)^{\alpha} F\right)^{\wedge}=(-i)^{m} \widehat{\lambda_{\alpha q}}$, we see that $\left(D^{\alpha} f\right)^{\wedge}=(-i)^{m}(2 \pi)^{n} \check{F}_{\alpha} \in$ $L_{\text {loc }}^{1}\left(\mathbf{R}^{n}\right)$, where $\check{F}_{c \alpha}(\xi)=F_{\alpha q}(-\xi)$. Let

$$
r(\xi)=\frac{1}{(2 \pi)^{2 n}|\xi|^{2 m} w(-\xi)},
$$

with $r(\xi)=\infty$ when $w(-\xi)=0$. If $d \rho(\xi)=r(\xi) d \xi$, then $\left(D^{\alpha} f\right)^{\wedge} \in L^{2}(\rho)$ and

$$
\left\|\left(D^{\prime \prime} f\right)^{\wedge}\right\|_{L^{2}(\rho)}^{2}=\int \frac{\xi^{2(x}|g(\xi)|^{2}}{|\xi|^{2 m}} d \mu(\xi) .
$$

Using (4.2) below with $l=m$,

$$
\sum_{|\propto|=m} \frac{m !}{\alpha !}\left\|\left(D^{\alpha} f\right)^{\wedge}\right\|_{L^{2}(\rho)}^{2}=\int|g|^{2} d \mu=(f, f)_{h} .
$$

Corollary 3.3. Let $m, h, \mu$, and $a_{*}$ be as in Theorem 2.1. Assume $d \mu(\xi)=$ $w(\xi) d \xi$ and $a_{i,}=0$ for all $|\gamma|=2 m$. Let $\rho$ be the Borel measure on $\mathbf{R}^{n}$ defined by $d \rho(\xi)=r(\xi) d \xi$, with $r$ as in (3.8). Then $f \in \mathscr{C}_{h, m}$ if and only if $f \in \mathscr{P}^{\prime}$ and $\left(D^{\prime \prime} f\right)^{\wedge} \in L^{2}(\rho)$ for every $|\alpha|=m$. In that case, $(f, f)_{h}$ is given by (3.9).

The translation invariant nature of $\mathscr{C}_{h, m}$ is evident in the following

Proposition 3.4. Let $\tau$ be a compactly supported Radon measure on $\mathbf{R}^{n}$. If $f$ is in $\mathscr{C}_{h, m}$, then so is $\tau * f$. Furthermore, if $\Lambda: \mathscr{C}_{h, m} \rightarrow L^{2}(\mu) \oplus H_{A}$ is as defined above and $\Lambda f=g \oplus\left(v+N_{A}\right)$, then $\Lambda(\tau * f)=\operatorname{tg} \oplus\left(t(0) v+N_{A}\right)$, where $t(\xi)=\int e^{i\langle x, \xi\rangle} d \tau(x)$.

Proof. If $\psi(x)=\int \varphi(x+y) d \tau(y)$, then $\langle\tau * f, \varphi\rangle=\langle f, \psi\rangle$ and

$$
\begin{aligned}
\widehat{\psi}(\xi) & =\iint e^{-i\langle x \cdot \xi\rangle} \varphi(x+y) d x d \tau(y) \\
& =\iint e^{-i\langle z-y \cdot \xi\rangle} \varphi(z) d z d \tau(y)=\widehat{\varphi}(\xi) t(\xi) .
\end{aligned}
$$


If $\Lambda f=g \oplus\left(v+N_{A}\right)$, so that (3.5) holds, then for all $\varphi \in \mathscr{D}_{m}$

$$
\begin{aligned}
\langle\tau * f, \varphi\rangle & =\int \widehat{\psi} g d \mu+\sum_{|\alpha|=m} D^{\alpha} \widehat{\psi}(0)(A v)_{\alpha} \\
& =\int \widehat{\varphi} \operatorname{tg} d \mu+\sum_{|\alpha|=m} t(0) D^{\alpha} \widehat{\varphi}(0)(A v)_{\alpha} .
\end{aligned}
$$

This gives (3.5), with $f, g, v$ replaced by $\tau * f, t g, t(0) v$; the assertions made are now apparent.

In the next result, (3.11) is equivalent to $\Lambda(\nu * h)=n \oplus\left(w+N_{A}\right)$ and (3.12) says $\nu(\bar{f})=(\nu * h, f)_{h}$. From this it is clear that $\mathscr{C}_{h, m}$ satisfies condition (c) in Theorem 1.1 of [11]. That conditions (a) and (b) are also satisfied can be seen from the discussion above in which the map $\Lambda$ was introduced. Applying Theorem 1.1 of [11], we conclude that $\mathscr{C}_{h, m}=C_{h}$.

Proposition 3.5. Let $m, h, \mu$ and $a_{\gamma}$ be as in Theorem 2.1. Let $\nu$ be a compactly supported Radon measure on $\mathbf{R}^{n}$ and assume that $\int x^{\alpha} d \nu(x)=0$ for all $|\alpha|<m$. Then $\nu * h \in \mathscr{C}_{h, m}$ and for all $\varphi$ in $\mathscr{D}_{m}$

$$
\langle\nu * h, \varphi\rangle=\int \widehat{\varphi} n d \mu+\sum_{|\alpha|=m}(A w)_{\alpha} D^{\alpha} \widehat{\varphi}(0),
$$

where $n(\xi)=\int e^{i\langle x, \xi\rangle} d \nu(x)$ and $w_{\beta}=D^{\beta} n(0)=\int(i x)^{\beta} d \nu(x)$. Furthermore, if $f \in \mathscr{C}_{h, m}$ and $\Lambda f=g \oplus\left(v+N_{A}\right)$, then

$$
\int \overline{f(x)} d \nu(x)=\int n \bar{g} d \mu+w^{T} \overline{A v} .
$$

Proof. If $\psi(z)=\int \varphi(z+y) d \nu(y)$, then from (2.4),

$$
\langle\nu * h, \varphi\rangle=\langle h, \psi\rangle=\int \widehat{\psi}-\widehat{\chi} T^{2 m-1} \widehat{\psi} d \mu+\sum_{|\eta| \leq 2 m} D^{\gamma} \widehat{\psi}(0) \frac{a_{\gamma}}{\gamma !}
$$

and, as in (3.10), $\widehat{\psi}=\widehat{\varphi} n$. Clearly, $D^{\alpha} n(0)=0$ for all $|\alpha|<m$. If $\varphi \in \mathscr{D}_{m}$, then $D^{\prime \prime} \widehat{\psi}(0)=0$ for $|\gamma|<2 m$, and for $|\gamma|=2 m$

$$
D^{\prime} \widehat{\psi}(0)=\sum_{\alpha+\beta=\gamma^{\prime}} \frac{\gamma !}{\alpha ! \beta !} D^{\alpha} \widehat{\varphi}(0) w_{\beta} .
$$

Thus, (3.11) follows from (3.13). To establish (3.12), choose a real-valued function $r$ in $\mathscr{D}$ with $\widehat{r}(0)=1$, and for $\varepsilon>0$ let $\overline{\varphi_{\varepsilon}(x)}=\int \varepsilon^{-n} r\left(\frac{x-y}{\varepsilon}\right) d \nu(y)$. Then $\varphi_{\varepsilon} \in \mathscr{D}_{m}$ and

$$
\left\langle f, \varphi_{\varepsilon}\right\rangle=\int \widehat{\varphi_{\varepsilon}} g d \mu+\sum_{|\alpha|=m}(A v)_{\alpha} D^{\alpha} \widehat{\varphi_{\varepsilon}}(0) .
$$

This yields (3.12) because

$$
\int \overline{f(x)} d \nu(x)=\lim _{\varepsilon \rightarrow 0} \overline{\left\langle f, \varphi_{\varepsilon}\right\rangle} \text { and } \widehat{\varphi_{\varepsilon}}(\xi)=\widehat{r}(\varepsilon \xi) \overline{n(\xi)} \text {. }
$$


For $s$ as in (1.1) we have $s=\nu * h$ with $\int \varphi d \nu=\sum_{i=1}^{N} c_{i} \varphi\left(x_{i}\right)$. Thus, such functions $s$ belong to $\mathscr{C}_{h, m}$.

The distribution $D^{\kappa} h,|\kappa| \geq m$, can be obtained as a limit of $\nu * h$ 's by choosing $\nu$ 's that correspond to appropriate difference operators. Such $\nu$ 's satisfy the orthogonality condition $\int x^{\alpha} d \nu(x)=0,|\alpha|<m$. Hence, the following may be regarded as a limiting case of the situation considered above.

Proposition 3.6. Let $m, h, \mu$ and $a_{\gamma}$, be as in Theorem 2.1. Fix $\kappa$ with $|\kappa| \geq m$ and let $p(\xi)=(i \xi)^{\kappa}$. Then, $p \in L^{2}(\mu)$ if and only if the distribution $D^{\kappa} h$ belongs to $\mathscr{C}_{h, m}$. In that case, $\Lambda\left((-D)^{\kappa} h\right)=p \oplus\left(w+N_{A}\right)$ with $w_{\alpha}=D^{\alpha} p(0)$, $|\alpha|=m$.

Proof. Let $\psi=D^{\kappa} \varphi$, so $\widehat{\psi}=p \widehat{\varphi}$. If $\varphi \in \mathscr{D}_{m}$, then, by a calculation like that for $(2.11)$,

$$
\sum_{|\gamma| \leq 2 m} D^{\gamma}(p \widehat{\varphi})(0) \frac{a_{\gamma}}{\gamma !}=\sum_{|\alpha|=m} \sum_{|\beta|=m} a_{\alpha+\beta} \frac{D^{\alpha} p(0)}{\alpha !} \frac{D^{\beta} \hat{\varphi}(0)}{\beta !} .
$$

Using (2.4), we have

$$
\left\langle(-D)^{\kappa} h, \varphi\right\rangle=\langle h, \psi\rangle=\int p \widehat{\varphi} d \mu+\sum_{|\beta|=m}(A w)_{\beta} D^{\beta} \widehat{\varphi}(0)
$$

for all $\varphi \in \mathscr{D}_{m}$. This is (3.5) with $f=(-D)^{\kappa} h, g=p$ and $v=w$. If $p \in$ $L^{2}(\mu)$ we apply Proposition 3.2 to see that $f \in \mathscr{C}_{h, m}$ and $\Lambda f=p \oplus\left(w+N_{A}\right)$. If $p \notin L^{2}(\mu)$ we apply Proposition 2.5 to obtain a sequence $\varphi_{i} \in \mathscr{D}_{2 k}$ such that $\int\left|\widehat{\varphi}_{i}\right|^{2} d \mu=1$ and $\int p \widehat{\varphi}_{i} d \mu \rightarrow \infty$. We take $2 k>m$ so that $D^{\beta} \widehat{\varphi}_{i}(0)=0$ when $|\beta|=m$. Then (3.14) gives

$$
\left\langle(-D)^{\kappa} h, \varphi_{i}\right\rangle=\int p \widehat{\varphi}_{i} d \mu \rightarrow \infty .
$$

Since $\left\|\widehat{\varphi}_{i}\right\|_{L^{2}(\mu)}^{2}+\left\|\hat{\varphi}_{i}^{(m)}(0)\right\|_{.}^{2}=1$, we see that $f=(-D)^{\kappa} h$ cannot satisfy (3.2) and hence cannot be in $\mathscr{C}_{h, m}$.

\section{ERROR ESTIMATES}

In this section we derive bounds on the difference between a function $g$ in $\mathscr{C}_{h, m}$ and a function $g^{X}$ of minimal $\mathscr{C}_{h, m}$ norm that agrees with $g$ on a set $X \subset \mathbf{R}^{n}$ of 'interpolation points'. These error estimates involve a parameter that measures the spacing of the points in $X$ and are of order $l$ in that parameter; our derivation assumes $l \geq m$ and

$$
\int|\xi|^{2 l} d \mu(\xi)<\infty
$$

For the examples given in $\S 5$, this assumption is satisfied for arbitrarily large values of $l$; see (5.2) below. In particular, the estimates apply to multiquadric interpolation, since the example there with $a=-1$ gives $h(x)=-2 \sqrt{\pi\left(1+|x|^{2}\right)}$. 
Before starting on the error estimates, we look at a related implication of (4.1). Let $p_{\alpha}(\xi)=(i \xi)^{\alpha}$. From

$$
\left(\xi_{1}^{2}+\cdots+\xi_{n}^{2}\right)^{l}=\sum_{|\alpha|=l} \frac{l !}{\alpha !} \xi^{2 \alpha}
$$

we observe that (4.1) holds if and only if $p_{\alpha} \in L^{2}(\mu)$ for all $|\alpha|=l$. If a distribution has all of its $l$ th order derivatives given by continuous functions, then it will belong to $C^{l}\left(\mathbf{R}^{n}\right)$. Thus, the following result shows that $(4.1)$ holds if and only if $\mathscr{C}_{h, m} \subset C^{l}\left(\mathbf{R}^{n}\right)$.

Proposition 4.1. Let $m, h, \mu$ and $a_{\gamma}$ be as in Theorem 2.1. Fix $\alpha$ with $|\alpha| \geq m$. Then the following are equivalent:

(a) $p_{\alpha} \in L^{2}(\mu)$, where $p_{\alpha}(\xi)=(i \xi)^{\alpha}$;

(b) for every $f$ in $\mathscr{C}_{h, m}$, the distribution $D^{\alpha} f$ belongs to $C\left(\mathbf{R}^{n}\right)$ and there is a constant $c_{\alpha}$ such that for all $f$ in $\mathscr{C}_{h, m},\left\|D^{\alpha} f\right\|_{\infty} \leq c_{\alpha}\|f\|_{h}$;

(c) there is a point $x_{0}$ in $\mathbf{R}^{n}$ and a constant $c_{\alpha}$ such that for all $f$ in $\mathscr{C}_{h, m} \cap C^{\infty},\left|D^{\alpha} f\left(x_{0}\right)\right| \leq c_{\alpha x}\|f\|_{h}$.

If these are true, then for all $f \in \mathscr{C}_{h, m}$ and all $y \in \mathbf{R}^{n}$,

$$
D^{\alpha} f(y)=\left(f, \delta_{y} *(-D)^{\alpha} h\right)_{h} .
$$

Proof. Let $f \in \mathscr{C}_{h, m}$ and let $F$ be its inverse Fourier transform, so that $\widehat{F}=f$. If $|\alpha|=m$, then, by Proposition 3.2, $\xi^{\alpha} F=\lambda_{\alpha}$ with $\lambda_{\alpha}$ given by (3.6). If $|\alpha|>m$, then $\alpha=\alpha^{\prime}+\beta$ with $\left|\alpha^{\prime}\right|=m$. Hence, $\xi^{\alpha} F=\lambda_{\alpha}$ with $\lambda_{\alpha}=\xi^{\beta} \lambda_{\alpha^{\prime}}$ where $\lambda_{\alpha^{\prime}}$ is given by (3.6). If (a) holds, then $\lambda_{\alpha}$ is finite; for $|\alpha|=m$, $\int d\left|\lambda_{\alpha}\right|=\int\left|\xi^{\alpha} g(\xi)\right| d \mu(\xi)+\left|(A v)_{\alpha}\right|$ and for $|\alpha|>m, \int d\left|\lambda_{\alpha}\right|=\int\left|\xi^{\alpha} g(\xi)\right| d \mu(\xi)$. Thus, $\widehat{\lambda}_{\alpha x}$ is continuous and bounded by $\int d\left|\lambda_{\alpha}\right|$. Since $(i D)^{\alpha} f=\left(\xi^{\alpha} F\right)^{\wedge}=\widehat{\lambda_{\alpha}}$, we see that (b) holds with $c_{\alpha}=\left\|p_{\alpha} \oplus\left(p_{\alpha}^{(m)}(0)+N_{A}\right)\right\|_{H}$. Thus, (a) implies (b).

That (b) implies (c) is obvious. To see that (c) implies (a), let $\psi$ be an arbitrary function in $\mathscr{D}\left(\mathbf{R}^{n} \sim\{0\}\right)$ and define $u$ by (2.8) with $\sigma=\psi \mu$ and $k=m$. Then, $u \in \mathscr{C}_{h, m}, \Lambda u=\psi \oplus 0$ and $\|u\|_{h}^{2}=\int|\psi|^{2} d \mu$. In addition, $u \in C^{\infty}$ and

$$
D^{\alpha} u\left(x_{0}\right)=\int e^{-i\left\langle x_{0}, \xi\right\rangle}(-i \xi)^{\alpha} \psi(\xi) d \mu(\xi)
$$

Thus, (c) gives $\left|\int e^{-i\left\langle x_{0}, \xi\right\rangle}(-i \xi)^{\alpha x} \psi(\xi) d \mu(\xi)\right| \leq c_{\alpha}\|\psi\|_{L^{2}(\mu)}$. Since this holds for all $\psi$ in $\mathscr{D}\left(\mathbf{R}^{n} \sim\{0\}\right)$, a dense subset of $L^{2}(\mu)$, (a) must be true.

To verify the last assertion, suppose $f \in \mathscr{C}_{h, m}$ with $\Lambda f=g \oplus\left(v+N_{A}\right)$. By Proposition 3.6, $\Lambda\left((-D)^{\alpha} h\right)=p_{\alpha} \oplus\left(p_{c}^{(m)}(0)+N_{A}\right)$. Using Proposition 3.4 with $\tau=\delta_{y}$, we have $t(\xi)=e^{i\langle y, \xi\rangle}$ and

$$
\Lambda\left(\delta_{y} *(-D)^{\alpha} h\right)=t p_{\alpha} \oplus\left(p_{\alpha}^{(m)}(0)+N_{A}\right) .
$$


Thus, $\left(f, \delta_{y} *(-D)^{\alpha} h\right)_{h}=\int g \overline{t p_{\alpha}} d \mu+v^{T} \overline{A p_{\alpha}^{(m)}(0)}=(-i)^{m} \widehat{\lambda_{\alpha}}(y)$. Here, $\lambda_{\alpha}$ is as above so, as already noted, $\widehat{\lambda_{\alpha}}=(i D)^{\alpha} \widehat{f}$; this gives the desired equality.

Our error estimates will be based on the following

Theorem 4.2. Let $m, h, \mu$ and $a_{\gamma}$ be as in Theorem 2.1. Assume that $\mu$ satisfies (4.1) with $l \geq \max \{1, m\}$. For a point $x_{0}$ in $\mathbf{R}^{n}$ suppose that $\sigma$ is a real-valued, compactly supported Radon measure on $\mathbf{R}^{n}$ such that

$$
p\left(x_{0}\right)=\int p(x) d \sigma(x)
$$

for all $p$ in $P_{l-1}$. Then for all $f$ in $\mathscr{C}_{h, m}$,

$$
\left|f\left(x_{0}\right)-\int f(x) d \sigma(x)\right| \leq c\|f\|_{h} \int\left|x-x_{0}\right|^{l} d|\sigma|(x)
$$

where $c=\left\{s+\int|\xi|^{2 l} /(l !)^{2} d \mu(\xi)\right\}^{1 / 2}$ with $s=\sum_{|\alpha|=m} \sum_{|\beta|=m}\left|A_{\alpha, \beta}\right|$ for $l=m$ and $s=0$ for $l>m$.

Proof. Let $\nu=\delta_{x_{0}}-\sigma$. By (4.4), $\int p(x) d \nu(x)=0$ for all $p \in P_{l-1}$. Since $l \geq m$, Proposition 3.5 applies to $\nu$, and from (3.12),

$$
\left|\int \overline{f(x)} d \nu(x)\right| \leq\left\|n \oplus\left(w+N_{A}\right)\right\|_{H}\|f\|_{h} .
$$

Here, $w_{\beta}=\int(i x)^{\beta} d \nu(x),|\beta|=m$. If $l>m$, then $w=0$; if $l=m$, then

$$
w_{\beta}=i^{m} \int\left(x-x_{0}\right)^{\beta} d \nu(x)=0-i^{m} \int\left(x-x_{0}\right)^{\beta} d \sigma(x) \text {. }
$$

Defining $R(\theta)$ by $e^{i \theta}=\sum_{k=0}^{l-1}(i \theta)^{k} / k !+R(\theta)$, we have $|R(\theta)| \leq|\theta|^{l} / l$ ! and

$$
\begin{aligned}
e^{-i\left\langle x_{0}, \xi\right\rangle} n(\xi) & =\int e^{i\left\langle x-x_{0}, \xi\right\rangle} d \nu(x)=\int R\left(\left\langle x-x_{0}, \xi\right\rangle\right) d \nu(x) \\
& =-\int R\left(\left\langle x-x_{0}, \xi\right\rangle\right) d \sigma(x) .
\end{aligned}
$$

If $b=\int\left|x-x_{0}\right|^{l} d|\sigma|(x)$, then $|n(\xi)| \leq b|\xi|^{l} / l$ ! and, for $l=m,\left|w_{\beta}\right| \leq b$. From this we obtain $\left\|n \oplus\left(w+N_{A}\right)\right\|_{H} \leq c b$ and (4.5) follows.

To obtain the error estimates mentioned at the beginning of this section, we apply Theorem 4.2 to $f=g-g^{X}$. Because of the minimum norm property of $g^{X},\|f\|_{h} \leq\|g\|_{h}$. Since other fixed bounds on $\|f\|_{h}$ result in acceptable error estimates, the minimum norm requirement on $g^{X}$ could be relaxed to simply a requirement that $\left\|g^{X}\right\|_{h}$ not exceed some set bound. If we choose $\sigma$ so that supp $\sigma \subset X$, then $\int g-g^{X} d \sigma=0$, and (4.5) gives

$$
\left|g\left(x_{0}\right)-g^{x}\left(x_{0}\right)\right| \leq c\|f\|_{h} \int\left|x-x_{0}\right|^{l} d|\sigma|(x) \text {. }
$$


To make such a choice of $\sigma$ possible, it may be necessary to restrict $x_{0}$. From (4.4) we see that if $p \equiv 0$ on supp $\sigma$ then $p\left(x_{0}\right)=0$. Let

$$
\begin{aligned}
N_{l-1}(X) & =\left\{p \in P_{l-1}: p(x)=0 \text { for all } x \in X\right\}, \\
\langle X\rangle_{l-1} & =\left\{x \in \mathbf{R}^{n}: p(x)=0 \quad \text { for all } p \in N_{l-1}(X)\right\} .
\end{aligned}
$$

Proposition 4.3. Let $E_{l-1}\left(x_{0}, X\right)$ be the set of all real-valued, compactly supported Radon measures on $\mathbf{R}^{n}$ that satisfy both (4.4) and $\operatorname{supp} \sigma \subset X$. Then $E_{l-1}\left(x_{0}, X\right)$ is nonempty if and only if $x_{0} \in\langle X\rangle_{l-1}$.

Proof. Necessity of $x_{0} \in\langle X\rangle_{l-1}$ is evident from the preceding discussion. To see that this is also sufficient, consider the linear functionals on $P_{l-1}$ defined by $L_{x}(p)=p(x)$. Choose a (finite) subset $Y$ of $X$ such that $\left\{L_{y}: y \in Y\right\}$ is linearly independent and $L_{x} \in \operatorname{span}\left\{L_{y}: y \in Y\right\}$ for all $x$ in $X$. Then, $N_{l-1}(Y)=N_{l-1}(X)$ and $\langle Y\rangle_{l-1}=\langle X\rangle_{l-1}$. Also, $\left\{L_{y}: y \in Y\right\}$ is a basis for $\left(P_{l-1} / N_{l-1}(Y)\right)^{\prime}$; let $\left\{p_{y}+N_{l-1}(Y): y \in Y\right\}$ be the dual basis. If the polynomials $p_{y}$ are replaced by their real parts, the result is still dual to $\left\{L_{y}: y \in Y\right\}$. We may therefore assume that each $p_{y}$ is real-valued. For $x_{0}$ in $\langle Y\rangle_{l-1}$, $L_{x_{0}}$ gives a linear functional on $P_{l-1} / N_{l-1}(Y)$. Thus, $L_{x_{0}}=\sum_{y \in Y} c_{y} L_{y}$, with $c_{y}=L_{x_{0}}\left(p_{y}\right)$, and it follows that $\sigma=\sum_{y \in Y} c_{y} \delta_{y}$ is in $E_{l-1}\left(x_{0}, X\right)$.

Of course, (4.7) will give a better error estimate if $\sigma$ is chosen from $E_{l-1}\left(x_{0}, X\right)$ so as to minimize $\int\left|x-x_{0}\right|^{l} d|\sigma|(x)$; we made no attempt to do this with our choice of $\sigma$ in the preceding proof.

We turn now to an analysis of the rate at which the error estimate goes to zero as the coverage by $X$ improves. For this we fix a region $\Omega$ and a function $g \in \mathscr{C}_{h, m}$ and, for various $X$, look at bounds on $\left|g-g^{X}\right|_{\Omega}$ given by (4.7). Here we use the notation $|f|_{\Omega}=\sup _{x \in \Omega}|f(x)|$.

The number $d=d(\Omega, X)$ defined by

$$
d(\Omega, X)=\sup _{y \in \Omega} \inf _{x \in X}|y-x|
$$

is a standard measurement of how closely $X$ covers $\Omega$. Using (4.7) and some mild assumptions about $\Omega$, we will show that

$$
\left|g-g^{X}\right|_{\Omega}=O\left(d^{l}\right)
$$

In order to use (4.7), we assume (4.1). In that case, Proposition 4.1 assures us of a uniform bound for the $l$ th order derivatives of $g-g^{X}$. From this and (4.9), we can deduce that the derivatives $D^{\prime \prime}\left(g-g^{X}\right)$ of intermediate order $0<|\alpha|<l$ satisfy $O\left(d^{l-|\alpha|}\right)$ estimates.

To establish (4.9), we proceed along lines used by Duchon [6]. We start by assuming that there are positive constants $M, \varepsilon_{0}$ such that for every $0<\varepsilon<$ $\varepsilon_{0}$,

$$
\Omega \subset \bigcup\left\{B(t, \varepsilon M): t \in T_{\varepsilon}\right\}
$$


where $T_{\varepsilon}=\left\{t \in \mathbf{R}^{n}: B(t, \varepsilon) \subset \Omega\right\}, B(t, r)=\left\{x \in \mathbf{R}^{n}:|x-t| \leq r\right\}$. Arguments in $\S 1$ of [6] show that such constants $M, \varepsilon_{0}$ will exist if $\Omega$ satisfies a cone condition.

Next we select a $P_{l-1}$-unisolvent set of points $\mathbf{a}(\alpha) \in \mathbf{R}^{n},|\alpha|<l$. A corresponding set of Lagrange polynominals, $p_{\gamma}^{\mathbf{a}} \in P_{l-1},|\gamma|<l$, is determined by the requirements: $p_{\gamma,}^{\mathbf{a}}(\mathbf{a}(\alpha))=1$, for $\alpha=\gamma ; p_{\gamma}^{\mathbf{a}}(\mathbf{a}(\alpha))=0$, for $\alpha \neq \gamma$. The matrix $A_{\alpha, \beta}=(\mathbf{a}(\alpha))^{\beta},|\alpha|<l,|\beta|<l$ is nonsingular. If $p_{\gamma,}(x)=\sum_{|\beta|<l}\left(A^{-1}\right)_{\beta, \gamma} x^{\beta}$, then $p_{\gamma, \gamma}(\mathbf{a}(\alpha))=\left(A A^{-1}\right)_{\alpha, \gamma}$, so $p_{\gamma}=p_{\gamma}^{\mathbf{a}}$. The function $\alpha \rightarrow \mathbf{a}(\alpha)$ can be identified with a point in $\mathbf{B}=\prod_{|\alpha|<l} B(\mathbf{a}(\alpha), \delta)$. Clearly, $\mathbf{b} \in \mathbf{B}$ if and only if $|\mathbf{b}(\alpha)-\mathbf{a}(\alpha)|<\delta$ for all $|\alpha|<l$. Now choose $\delta>0$ so that $B_{\alpha, \beta}=(\mathbf{b}(\alpha))^{\beta}$ is invertible for all $\mathbf{b} \in \mathbf{B}$. As justified by replacing the points $\mathbf{a}(\alpha)$ with the points $\delta^{-1} \mathbf{a}(\alpha)$, we assume $\delta=1$.

Choose $R$ so that $B(0, R)$ contains all the unit balls $B(\mathbf{a}(\alpha), 1),|\alpha|<l$. The Lagrange polynomials $p_{c r}^{\mathbf{b}}$ depend continuously on $\mathbf{b}$. Let

$$
\lambda(r)=\sup \left\{\sum_{|\alpha|<l}\left|p_{\alpha}^{\mathbf{b}}(x)\right|:|x| \leq r, \mathbf{b} \in \mathbf{B}\right\} .
$$

For $d=d(\Omega, X)<\varepsilon_{0} / R$, set $\varepsilon=R d$ and fix a point $t$ in $T_{\varepsilon}$. The balls $B(t+d \mathbf{a}(\alpha), d)$ are contained in $B(t, R d)=B(t, \varepsilon) \subset \Omega$. By (4.8), for every $|\alpha|<l$, there is at least one point $x_{\alpha}$ in $X \cap B(t+d \mathbf{a}(\alpha), d)$. If $\mathbf{b}$ is the point in B defined by $x_{c}=t+d \mathbf{b}(\alpha)$, and

$$
\sigma=\sum_{|x|<l} p_{c}^{\mathbf{b}}\left(\frac{x_{0}-t}{d}\right) \delta_{x_{a}}
$$

with $x_{0}$ arbitrary, then supp $\sigma \subset X \cap B(t, \varepsilon)$, and (4.4) holds for all $p \in P_{l-1}$; to verify (4.4), take $q$ so that $p(x)=q((x-t) / d)$ and use $\sum_{|x|<l} p_{\alpha}^{\mathbf{b}}(y) q(\mathbf{b}(\alpha))=$ $q(y)$ with $y=\left(x_{0}-t\right) / d$.

Suppose $x_{0} \in B(t, \varepsilon M+d)$. Then, $\left|x_{0}-t\right| / d \leq(R M+1)$, so $\int d|\sigma| \leq$ $\lambda(R M+1)$. Also, for $x \in \operatorname{supp} \sigma$,

$$
\left|x-x_{0}\right| \leq|x-t|+\left|t-x_{0}\right| \leq(R+R M+1) d .
$$

Thus, $\int\left|x-x_{0}\right|^{l} d|\sigma| \leq C^{0} d^{l}$ with $C^{0}=(R+R M+1)^{l} \lambda(R M+1)$. Since $x_{0}$ is any point in $B(t, \varepsilon M+d)$, (4.7) gives $\left|g-g^{X}\right|_{B(t, \varepsilon M+d)} \leq c\|f\|_{h} C^{0} d^{l}$. By (4.10), if $y \in \Omega$, we can choose $t \in T_{\varepsilon}$ so that $y \in B(t, \varepsilon M)$. Then $B(y, d) \subset B(t, \varepsilon M+d)$, so for every $y \in \Omega$,

$$
\left|g-g^{x}\right|_{B(y, d)} \leq c C^{0}\|f\|_{h} d^{\prime} .
$$

This is more than required for (4.9), but will be useful for derivative estimates.

By Proposition 4.1, $f=g-g^{X}$ is in $C^{l}\left(\mathbf{R}^{n}\right)$. For $y \in \Omega, \theta \in \mathbf{R}$ and $u \in \mathbf{R}^{n}$ with $|u|=1$, let $\varphi(\theta)=f(y+\theta u)$. Then

$$
\varphi^{(k)}(\theta)=k ! \sum_{|a|=k} \frac{u^{(k}}{\alpha !} D^{\prime k} f(y+\theta u) .
$$


By (b) in Proposition (4.1), $\left|\varphi^{(l)}\right|_{\mathbf{R}} \leq C^{\prime}\|f\|_{h}$ with $C^{\prime}=l ! \sum_{|\alpha|=l} c_{\alpha} / \alpha$ !. From (4.11) we also have a bound on $|\varphi|_{I}$ where $I$ is the interval $[-d, d]$. For $0<k<l$, the results of Gorny [8] summarized in [12] then give

$$
\left|\varphi^{(k)}(0)\right| \leq C_{k}\|f\|_{h} d^{l-k},
$$

where $C_{k}=16(2 e)^{k}\left(c C^{0}\right)^{1-k / l}\left[\max \left(C^{\prime}, l ! 2^{-l} c C^{0}\right)\right]^{k / l}$. Note that $C_{k}$ can be calculated from $n, l, m, h$ and $M$; the choice of $R$ depends only on $l$ and $n$, so $C^{0}$ requires only $l, n, M$, while $c$ and $C^{\prime}$ require only $m, h, l, n$. Combining (4.12) and (4.13) gives

$$
\sup _{|u|=1}\left|\sum_{|\alpha|=k} \frac{u^{\alpha}}{\alpha !} D^{\alpha} f(y)\right| \leq \frac{C_{k}}{k !}\|f\|_{h} d^{l-k}
$$

for every $y \in \Omega$. Since

$$
|v|_{k}=\sup _{|u|=1}\left|\sum_{|\alpha|=k} \frac{u^{\alpha}}{\alpha !} v_{\alpha}\right|
$$

is a norm for $V_{k}$, we conclude that $\left|D^{\alpha} f\right|_{\Omega}=O\left(d^{l-|\alpha|}\right)$ for every $|\alpha| \leq l$. To summarize, we state

Theorem 4.4. Let $m, h, \mu$ and $a_{\gamma}$ be as in Theorem 2.1. Assume (4.1) holds with $l \geq \max \{1, m\}$, and suppose $\Omega$ is a subset of $\mathbf{R}^{n}$ that satisfies (4.10) for some $\bar{M}, \varepsilon_{0}>0$. Then there are positive constants $C, d_{0}$ such that if $f \in \mathscr{C}_{h, m}$ vanishes on a set $X$ and the number $d=d(\Omega, X)$ defined by (4.8) is less than $d_{0}$, then for all $|\alpha| \leq l$,

$$
\left|D^{\alpha} f\right|_{\Omega} \leq C\|f\|_{h} d^{l-|\alpha|}
$$

\section{EXAMPLES}

In this section we look at some examples of conditionally positive definite functions $h$. For these examples we determine the measure $\mu$ and coefficients $a_{\eta,},|\gamma|=2 m$, that appear in (2.4). As can be seen from (5.2) below, these examples all satisfy (4.1) and do so for arbitrarily large choices of $l$. Thus the error estimates in $\S 4$ apply, showing that for interpolation based on any of the $h$ 's given here, approximation of arbitrarily high order can be achieved.

For $a \in \mathbf{R}$, let $w_{a}$ be the function on $\mathbf{R}^{n}$ defined by

$$
w_{a}(\xi)=\frac{2 K_{(n-a) / 2}(|\xi|)}{(2 \pi)^{n / 2} 2^{a / 2}|\xi|^{(n-a) / 2}},
$$

where $K_{\nu}$, is a modified Bessel function of the second kind. From the behavior of $K_{\nu}(r)$ at $r=0$ and $r=\infty$ we note that

$$
\int|\xi|^{2 l} w_{a}(\xi) d \xi<\infty
$$


if and only if $a+2 l>0$. For $a \in \mathbf{R}, a \neq 0,-2,-4, \ldots$, let

$$
h_{a}(x)=\frac{\Gamma(a / 2)}{\left(1+|x|^{2}\right)^{a / 2}}
$$

and for $a=-2 k, k=0,1,2, \ldots$, define $h_{a}$ by

$$
\begin{aligned}
h_{-2 k}(x) & =\lim _{a \rightarrow-2 k}\left[h_{a}(x)-\Gamma(a / 2)\left(1+|x|^{2}\right)^{k}\right] \\
& =\frac{(-1)^{k+1}}{k !}\left(1+|x|^{2}\right)^{k} \log \left(1+|x|^{2}\right) .
\end{aligned}
$$

The last equality can be verified by using $\Gamma\left(\frac{a}{2}+k+1\right)=\left(\frac{a}{2}+k\right) \cdots\left(\frac{a}{2}\right) \Gamma\left(\frac{a}{2}\right)$ together with

$$
\left.\frac{d}{d t}\right|_{t=k}\left(1+|x|^{2}\right)^{t}=\lim _{a \rightarrow-2 k} \frac{\left(1+|x|^{2}\right)^{-a / 2}-\left(1+|x|^{2}\right)^{k}}{(-a / 2)-k} .
$$

Lemma 5.1. If $\widehat{\varphi} \in \mathscr{D}\left(\mathbf{R}^{n} \sim\{0\}\right)$, then for all $a$ in $\mathbf{R}$

$$
\int h_{a}(x) \varphi(x) d x=\int \widehat{\varphi}(\xi) w_{a}(\xi) d \xi .
$$

Proof. A basic fact used in the theory of Bessel potentials is that (5.5) holds for all $\varphi \in \mathscr{S}$ if $a>0$; see [2], [3] or [4]. For $\hat{\varphi} \in \mathscr{D}\left(\mathbf{R}^{n} \sim\{0\}\right)$ an analytic continuation argument gives $(5.5)$ for $a \neq 0,-2,-4, \ldots$ To obtain (5.5) for the remaining values of $a=-2 k$, we take limits. If $f(t)=\left(1+|x|^{2}\right)^{t}$ and $a \neq 0,-2,-4, \ldots$, then

$$
\left[h_{a}(x)-\Gamma\left(\frac{a}{2}\right)\left(1+|x|^{2}\right)^{k}\right]=\left(\frac{a}{2}+k\right) \Gamma\left(\frac{a}{2}\right) \int_{0}^{1} f^{\prime}\left(k-\left(\frac{a}{2}+k\right) s\right) d s .
$$

Estimates from this can be used to justify an application of Lebesgue's dominated convergence theorem that shows

$$
\int h_{-2 k}(x) \varphi(x) d x=\lim _{a \rightarrow-2 k} \int\left[h_{a}(x)-\Gamma\left(\frac{a}{2}\right)\left(1+|x|^{2}\right)^{k}\right] \varphi(x) d x .
$$

Now $\hat{\varphi} \in \mathscr{D}\left(\mathbf{R}^{n} \sim\{0\}\right)$, so $\int\left(1+|x|^{2}\right)^{k} \varphi(x) d x=0$. We therefore have $\int h_{-2 k}(x) \varphi(x) d x=\lim _{a \rightarrow-2 k} \int \widehat{\varphi}(\xi) w_{a}(\xi) d \xi$, which gives $(5.5)$ for $a=-2 k$.

Theorem 5.2. If $m$ is a nonnegative integer and $a+2 m>0$, then (2.4) holds with $h=h_{a}, d \mu(\xi)=w_{a}(\xi) d \xi$, and $a_{\gamma}=0$ for $|\gamma|=2 m$.

Proof. If $m=0$, then $a>0$. As already mentioned, (5.5) holds for all $\varphi$ in $\mathscr{S}$ if $a>0$; thus, we have (2.4) with $m=0$ and $a>0$. For the rest of the proof we assume $m \geq 1$. Let

$$
u_{a}(x)=\int\left[e^{-i\langle x, \xi\rangle}-\widehat{\chi}(\xi) \sum_{k=0}^{2 m-1} \frac{(-i\langle x, \xi\rangle)^{k}}{k !}\right] w_{a}(\xi) d \xi
$$

By Proposition 2.2 we have $u_{a} \in C\left(\mathbf{R}^{n}\right), u_{a}(x)=o\left(|x|^{2 m}\right)$, and for all $\varphi$ in $\mathscr{S}$

$$
\int u_{a}(x) \varphi(x) d x=\left\langle S_{a}, \widehat{\varphi}\right\rangle
$$


where $\left\langle S_{a}, \psi\right\rangle=\int\left[\psi-\widehat{\chi} T^{2 m-1} \psi\right](\xi) w_{a}(\xi) d \xi$. Let $T_{a}$ be the tempered distribution defined by $\int h_{a}(x) \varphi(x) d x=\left\langle T_{a}, \widehat{\varphi}\right\rangle$. By $(5.5),\left\langle T_{a}, \psi\right\rangle=\left\langle S_{a}, \psi\right\rangle$ for all $\psi \in \mathscr{D}\left(\mathbf{R}^{n} \sim\{0\}\right)$. Thus, $\left(T_{a}-S_{a}\right)^{\uparrow}=h_{a}-u_{a}$ is a polynomial $q$. Both $h_{a}$ and $u_{a}$ are $o\left(|x|^{2 m}\right)$ at $|x|=\infty$, so $\operatorname{deg} q<2 m$. The desired instance of (2.4) now follows from $\left\langle h_{a}-q, \varphi\right\rangle=\left\langle S_{a}, \widehat{\varphi}\right\rangle$.

\section{Equivalence of Definitions}

Theorem 6.1 below, when combined with Proposition 2.4, shows the equivalence of the definition of conditional positive definiteness adopted here with that used in [11]. As in [11], we define $P_{m-1}^{\perp}$ to be the space of all finite measures $\nu$ on $\mathbf{R}^{n}$ that have support consisting of a finite set of points and satisfy $\nu(p)=0$ for all $p \in P_{m-1}$. The space obtained by relaxing the support requirement to allow compact sets, rather than only finite sets, will be denoted by $\left\langle P_{m-1}^{\perp}\right\rangle$. If $\nu=\sum_{i=1}^{N} c_{i} \delta_{x_{i}}$, then

$$
\nu(\overline{\nu * \bar{h}})=\sum_{i=1}^{N} \sum_{j=1}^{N} c_{i} \bar{c}_{j} h\left(x_{i}-x_{j}\right),
$$

and $\nu \in P_{m-1}^{\perp}$ if and only if $\sum_{i=1}^{N} c_{i} x_{i}^{(x}=0$ for all $|\alpha|<m$. If $d \nu(x)=\varphi(x) d x$ then

$$
\nu(\overline{\nu * \bar{h}})=\iint \varphi(x) \overline{\varphi(y)} h(x-y) d x d y,
$$

and $\nu$ is in $\left\langle P_{m-1}^{\perp}\right\rangle$ if $\varphi \in \mathscr{D}_{m}$.

Theorem 6.1. Let $h$ be an arbitrary function in $C\left(\mathbf{R}^{n}\right)$. If $\nu(\overline{\nu * \bar{h}}) \geq 0$ holds for all $\nu \in P_{m-1}^{\perp}$, then it holds for all $\nu \in\left\langle P_{m-1}^{\perp}\right\rangle$.

Proof. Fix $\nu$ in $\left\langle P_{m-1}^{\perp}\right\rangle$ and let $K$ be its support. Recall that the finite Borel measures on $K$ form the dual $C(K)^{\prime}$ of $C(K)$, the continuous functions on $K$ with the sup norm topology. The norms involved in this duality will be written as follows: for $f \in C(K),|f|_{K}=\sup _{x \in K}|f(x)|$; for $\sigma \in C(K)^{\prime},\|\sigma\|=\int d|\sigma|$. Let $h_{y}(x)=h(y-x) . K$ is compact, so for every $\varepsilon>0$ there is a finite set $F_{\varepsilon} \subset K$ such that, if $y \in K$, then $\left|h_{y}-h_{y_{0}}\right|_{K}<\varepsilon$ for at least one $y_{0} \in F_{\varepsilon}$. If $\sigma$ is in the weak* neighborhood

$$
U\left(\nu, F_{\varepsilon}, \varepsilon\right)=\left\{\sigma \in C(K)^{\prime}:\left|(\sigma-\nu)\left(\bar{h}_{y_{0}}\right)\right|<\varepsilon \text { for all } y_{0} \in F_{\varepsilon}\right\}
$$

and $y \in K$, then, for a suitable choice of $y_{0} \in F_{\varepsilon}$,

$$
\left|(\sigma-\nu)\left(\bar{h}_{y}\right)\right|=\left|(\sigma-\nu)\left(\bar{h}_{y}-\bar{h}_{y_{0}}\right)+(\sigma-\nu)\left(\bar{h}_{y_{0}}\right)\right| \leq(\|\sigma-\nu\|+1) \varepsilon .
$$

Since $(\sigma-\nu) * \bar{h}(y)=(\sigma-\nu)\left(\bar{h}_{y}\right)$, we get $|(\sigma-\nu) * \bar{h}|_{K} \leq(\|\sigma-\nu\|+1) \varepsilon$ for all $\sigma \in U\left(\nu, F_{\varepsilon}, \varepsilon\right)$. For such $\sigma$ let $w$ be the number defined by

$$
w=\sigma(\overline{\sigma * \bar{h}})-\nu(\overline{\nu * \bar{h}})=\sigma((\overline{\sigma-\nu) * \bar{h}})+(\sigma-\nu)(\overline{\nu * \bar{h}})
$$

and observe $|w| \leq\|\sigma\||(\sigma-\nu) * \bar{h}|_{K}+|(\sigma-\nu)(\overline{\nu * \bar{h}})|$. 
Let $B=\left\{\sigma \in C(K)^{\prime}:\|\sigma\| \leq\|\nu\|\right\}$ and take $C=B \cap\left\langle P_{m-1}^{\perp}\right\rangle, S=B \cap P_{m-1}^{\perp}$. By arguments given below, $S$ is weak* dense in $C$. This allows us to choose

$$
\sigma \in S \cap\left\{\sigma \in U\left(\nu, F_{\varepsilon}, \varepsilon\right):|(\sigma-\nu)(\overline{\nu * \bar{h}})|<\varepsilon\right\} .
$$

For that choice we have $\sigma(\overline{\sigma * \bar{h}}) \geq 0$ and

$$
|w| \leq\|\sigma\|(\|\sigma-\nu\|+1) \varepsilon+\varepsilon \leq\|\nu\|(2\|\nu\|+1) \varepsilon+\varepsilon .
$$

Since $w$ is arbitrarily small, we see that $\nu(\overline{\nu * \bar{h}})$ must be arbitrarily close to points on the positive real axis and hence must be greater than or equal to zero.

$C$ is convex and weak* compact so, by the Krein-Milman theorem, $C$ is the closed convex hull of its extreme points. Since $S$ is convex, it will be weak* dense if it contains all of the extreme points of $C$. Suppose $\sigma_{0}$ is an extreme point of $C$ that is not in $S$. Then supp $\sigma_{0}$ cannot be a finite set, so we can subdivide it into $J=2\left(1+\operatorname{dim} P_{m-1}\right)$ disjoint subsets $E_{1}, \ldots, E_{J}$ with $\left|\sigma_{0}\right|\left(E_{j}\right) \neq 0$. Let $\sigma_{j}(E)=\sigma_{0}\left(E_{j} \cap E\right)$ and take $c_{\alpha, j}=\int x^{\alpha} d \sigma_{j}(x)$. By a dimension argument, there is a point $a \in \mathbf{R}^{J} \sim\{0\}$ that satisfies the equations

$$
\sum_{j=1}^{J} a_{j}\left\|\sigma_{j}\right\|=0 ; \quad \sum_{j=1}^{J} a_{j} c_{\alpha, j}=0, \quad|\alpha|<m .
$$

For $t \in \mathbf{R}$, let $\sigma^{t}=\sum_{j=1}^{J}\left(1+t a_{j}\right) \sigma_{j}$. Then, $\sigma^{t} \in\left\langle P_{m-1}^{\perp}\right\rangle$, and if $\left(1+t a_{j}\right) \geq 0$,

$$
\left\|\sigma^{t}\right\|=\sum_{j=1}^{J}\left(1+t a_{j}\right)\left\|\sigma_{j}\right\|=\sum_{j=1}^{J}\left\|\sigma_{j}\right\|=\left\|\sigma_{0}\right\| \leq\|\nu\| .
$$

Thus, $\sigma^{t} \in C$ for all $t$ in an interval about 0 . This contradicts the assumption that $\sigma_{0}$ was an extreme point of $C$ because $\sigma^{t}=\sigma_{0}$ only if $t=0$, as seen from the fact that $a \neq 0$ and $\left\|\sigma_{j}\right\| \neq 0$ for all $j=1, \ldots, J$.

\section{BIBLIOGRAPHY}

1. J. H. Ahlberg, E. N. Nilson, and J. L. Walsh, The theory of splines and their applications, Academic Press, New York, 1967.

2. N. Aronszajn and K. T. Smith, Theory of Bessel potentials. I, Ann Inst. Fourier 11 (1961), 385-475.

3. A. P. Calderon, Lebesgue spaces of differentiable functions and distributions, Proc. Sympos. Pure Math., vol. 5, Amer. Math. Soc., Providence, R.I., 1961, pp. 33-49.

4. W. F. Donoghue, Distributions and Fourier transforms, Academic Press, New York, 1969.

5. J. Duchon, Splines minimizing rotation-invariant semi-norms in Sobolev spaces, in Constructive Theory of Functions of Several Variables (W. Schempp and K. Zeller, eds.), SpringerVerlag, Berlin-Heidelberg, 1977, pp. 85-100.

6. __ Sur l'erreur d'interpolation des fonctions de plusieurs variables par les $D^{m}$-splines, RAIRO Anal. Numér. 12 (1978), 325-334.

7. I. M. Gelfand and N. Ya. Vilenkin, Generalized functions, Vol. 4, Academic Press, New York, 1964.

8. A. Gorny, Contribution à l'étude des fonctions dérivables d'une variable réelle, Acta Math. 71 (1939), 317-358. 
9. J. L. Kelly and I. Namioka, Linear topological spaces, Van Nostrand, Princeton, NJ, 1963.

10. W. R. Madych and S. A. Nelson, Multivariate interpolation: a variational theory, manuscript, 1983.

11. Multivariate interpolation and conditionally positive definite functions, Approx. Theory and its Applications 4 (1988), no. 4, 77-89.

12. D. S. Mitrinović, Analytic inequalities, Springer-Verlag, Berlin-Heidelberg, 1970.

Department of Mathematics, University of Connecticut, Storrs, Connecticut 06268. E-mail:madych@uconnvm.bitnet

Department of Mathematics, Iowa State University, Ames, Iowa 50011 